\title{
Visible persistence following a brief increment in stimulus luminance
}

\author{
CRAIG D. CLARK and JOHN H. HOGBEN \\ The University of Western Australia, Nedlands, Australia
}

\begin{abstract}
In previous studies, it has been demonstrated that visible persistence-the period for which the perceived duration of a stimulus exceeds its physical duration-can be extended by briefly incrementing the luminance of the stimulus immediately prior to offset. Using a two-component pattern integration task, we show that this effect is not an artifact of change in the total luminous flux within the stimulus. Visible persistence was unaffected by overall luminance of the stimulus. It was also time-locked to the luminance increment. Visible persistence is seen to result from a process that is initiated by stimulus onset and that can be either wholly or partially reinitiated by the onset of the luminance increment. The duration of this process (which determines the duration of stimulus visibility) can be modified in a graded fashion by stimulus events that occur after its initiation. We outline a single-process inhibitory feedback model of the persistence mechanism that accounts for the present findings.
\end{abstract}

Visual displays presented for less than about $100 \mathrm{msec}$ remain visible for some interval after the display has physically terminated (Efron, 1970a, 1970b). The additional period of visibility is known as visible persistence (Coltheart, 1980).

Visible persistence is characterized by stimulus dependencies that differentiate it from other well-studied visual aftereffects such as retinal afterimages (Coltheart, 1980; Di Lollo, Clark, \& Hogben, 1988), and from well-known forms of short-term memory such as iconic memory (Coltheart, 1980). In particular, iconic memory and afterimages are known to increase in duration as the energy of the inducing stimulus is increased, by increments in either its luminance or its duration. By contrast, it is well established that the duration of visible persistence is inversely related to the duration of the stimulus (the inverse duration effect of Coltheart, 1980) and that visible persistence is usually inversely related to stimulus luminance (the inverse intensity effect).

Various general accounts of the mechanism of visible persistence have been proposed. Di Lollo and Bourassa (1983) outlined two that they regarded as equally tenable. The first of these-the processing hypothesis-derives from the sensory-coding hypothesis put forward by Di Lollo (1980). In essence, this holds that persistence is a product of the activity of sensory-coding mechanisms that operate at the earliest stages of visual processing to recode the retinal image in terms of primitive features such as edges, bars, dots, and discontinuities. According to the

This work was supported by an Australian Psychological Society Visiting Research Fellowship to C. D. Clark and by a grant from the Australian Research Council to John H. Hogben. The experimental work was completed while the authors were visiting the University of Alberta. Requests for reprints should be sent to John Hogben, Department of Psychology, The University of Western Australia, Nedlands, W.A. 6009, Australia. processing hypothesis, the duration of the activity that a stimulus initiates in the persistence mechanism corresponds to the duration of very early stages in a chain of processing events triggered by a change in visual stimulation. Stimuli persist to the extent that this activity outlasts the corresponding physical stimulation.

The second of the two accounts outlined by Di Lollo and Bourassa (1983) was proposed initially by Serviere, Miceli, and Galifret (1977a, 1977b) and subsequently elaborated by Coltheart (1980). It holds that the onset of the activity underlying persistence is time-locked to the first positive peak of the cortical on response elicited by stimulus onset, and that the termination of this activity is similarly time-locked to the cortical off response elicited by stimulus offset. The rationale for this hypothesis is founded largely on electrophysiological studies showing that, as the duration of an inducing stimulus increases towards approximately $60 \mathrm{msec}$, the latency of the cortical off response decreases to a minimum, asymptotic value (Serviere et al., 1977a): as a result, the time interval between on and off responses is approximately constant for brief stimuli, mirroring psychophysical findings. A theoretical link between the latency of the off response and the termination of persistence provides a straightforward explanation for the inverse relationship between the duration of persistence and the duration of the inducing stimulus.

These two accounts represent fundamentally different theoretical views of the mechanism of persistence. The fact that both have remained viable in the literature (e.g., Di Lollo \& Hogben, 1987) illustrates that theoretical development in this area is in need of a more complete empirical characterization of the persistence mechanism. One approach has been the examination of factors that either attenuate or prolong visible persistence.

Among the factors attenuating persistence, the spatial proximity of successive stimuli has received recent atten- 
tion. Farrell (1984), employing the display of rotating radial lines investigated by Dixon and Hammond (1972) and Efron and Lee (1971), found that duration of persistence increased with the angular separation between successive lines. In a similar study, Di Lollo and Hogben (1985) clarified the effects of separation between successive stimuli by using points of light rather than lines. They concluded that the visible persistence of a stimulus was suppressed by another stimulus presented nearby and soon after, and that the degree of suppression was directly related to the proximity of the stimuli. They also found that both the duration of visible persistence and the magnitude of suppression increased with retinal eccentricity. These authors have further demonstrated the spatial proximity effect in an apparent motion display (Hogben \& Di Lollo, 1985) and in a display designed to exclude or at least minimize apparent motion (Di Lollo \& Hogben, 1987), at the same time demonstrating the importance of stimulus and background luminance in facilitating the suppression of visible persistence. The dependence of suppression on the proximity of stimuli, on retinal location, and on luminance has suggested the involvement of inhibition at some unidentified level within the visual system.

Somewhat less attention has been given to stimulus conditions under which visible persistence is prolonged. Two stimulus manipulations have been reported to reinitiate or extend the persistence of long-lasting visual displays. Di Lollo (1980, Experiment 2) found that such displays were given a new lease of persistence by a small spatial translation of individual pattern elements. Observers were presented with displays made up of multiple three-point clusters, in which the points in each cluster marked the corners of a triangle. These displays persisted longer when the triangles were inverted for the last $10 \mathrm{msec}$ of the display interval. A similar effect was obtained by Di Lollo and Bourassa (1983), who found that the persistence of displays as long as $200 \mathrm{msec}$ was increased when they simply incremented the luminance of the display for the last $20 \mathrm{msec}$.

Di Lollo and Bourassa's (1983) findings are potentially of considerable importance in characterizing the persistence mechanism, in that they apparently show persistence to be prolonged by a stimulus manipulation that neither alters the pattern information content of the display nor involves the movement of display elements to previously unstimulated retinal locations. These results have been interpreted as showing that the luminance increment acts to reinitiate activity in the persistence mechanism. However, it is not entirely clear that the longer persistence produced by briefly incrementing display luminance was not due, instead, to the accompanying increase in the total luminous flux in the display.

Although, as noted above, persistence is typically found to be inversely related to stimulus luminance (and, therefore, to total luminous flux), a direct relationship is obtained under conditions that have yet to be adequately characterized (Nisly \& Wasserman, 1989). Di Lollo and Bourassa (1983) go some way toward addressing the pos- sible confounding of increment presentation with change in total luminous flux in their third experiment, in which the luminance of a brief $(20$-msec) stimulus is shown to have virtually no effect on its persistence. However, it cannot be automatically concluded that the same would be true of the longer inducing stimuli, for which the brief luminance increment had its greatest effect. The persistence of very brief displays and that of long-lasting displays are known to differ in their response to a range of stimulus parameters (Lovegrove, Martin, \& Slaghuis, 1986), leading to speculation that the process or processes mediating persistence change as a function of stimulus duration (e.g., Bowling \& Lovegrove, 1980).

In addition, stimuli that are sustained during the relatively long-latency photochemical component of the visual on response (Baker, 1963) may be more effective than very brief stimuli at eliciting retinal afterimages. As noted by Breitmeyer (1984), afterimages produced by very highcontrast displays can generate a direct relationship between the duration of stimulus visibility and total luminous flux. Di Lollo et al. (1988) have demonstrated that observers can use such afterimages to perform the pattern integration task employed by Di Lollo and Bourassa (1983) to measure persistence.

The second aspect of Di Lollo and Bourassa's (1983) study that admits doubt concerning the manner in which the luminance increment acts to extend persistence is the absence of any direct evidence that the additional period of persistence bears a strict temporal relationship to the brief luminance increment. If the luminance increment does initiate an additional period of persistence, this period should be time-locked to the onset of the increment. On the other hand, if the increment acts in combination with the remainder of the display, whether by incrementing total luminous flux or perhaps supplementing ongoing activity in some way, persistence should remain time-locked to the original onset of the stimulus.

In the present study, we reexamined the effect of a brief luminance increment on the persistence of a long-lasting display, addressing both the effect of total luminous flux and the temporal relationship between persistence and the luminance increment. We used the two-component pattern integration task employed by Di Lollo and Bourassa (1983), which has been used extensively in the study of visible persistence. The rationale for this task is as follows. When two brief stimuli are presented in sufficiently rapid succession, the period for which the first stimulus persists will overlap the presentation of the second stimulus, so that the two stimuli will be simultaneously visible for some interval (i.e., be temporally integrated). The longer the persistence of the first stimulus, the longer will be the duration of overlap between the periods of visibility of the two stimuli. The duration of this overlap is assumed to be directly related to performance on tasks that require an observer to respond to some feature of the composite pattern formed when the two stimuli are temporally integrated. Persistence is estimated through adjustment of the interval that separates the first and second stimuli 
until a constant duration of perceptual overlap is obtained (i.e., until observers reach some criterion level of performance on the temporal integration task). The separation interval required to produce constant overlap in this way is an index of the persistence of the first stimulus.

Using the two-component pattern integration task, we measured the persistence of a long-lasting stimulus of fixed duration $(100 \mathrm{msec})$ in which a brief luminance increment was presented at one of a number of temporal positions ranging from the beginning to the end of the stimulus. In this case, the total luminous flux in the stimulus was independent of the temporal position of the luminance increment. We found the duration of persistence to vary in a near-linear fashion with the temporal position of the luminance increment within the stimulus, with persistence increasing monotonically as the increment moved progressively closer to the end of the stimulus. These results substantiate Di Lollo and Bourassa's (1983) key inferences that the increment acts to reinitiate a period of activity in the persistence mechanism and that the increment itself, rather than the associated increase in total luminous flux, is responsible for the increased duration of persistence.

The present study also extends previous research in showing that the increase in persistence observed when a brief luminance increment is presented at the end of a long-lasting display is one aspect of a more complex pattern of results that also includes a reduction in persistence when this increment is presented at the beginning of the stimulus, and no effect when the luminance of the stimulus is incremented for its entire duration.

We present a simple model of a persistence mechanism with the functional properties necessary to accommodate the effects observed.

\section{EXPERIMENT 1}

In Experiment 1, we varied the temporal position of a brief luminance increment within a long-lasting first stimulus of constant duration. In this way, we were able to vary the interval separating the luminance increment from the second stimulus without changing the total luminous flux within the first stimulus.

If a brief luminance increment does reinitiate activity in the persistence mechanism, then the degree of perceptual integration will vary with the interval separating the onset of the luminance increment from that of the second stimulus. This interval is referred to here as the effective stimulus onset asynchrony, or ESOA, to distinguish it from the conventional stimulus onset asynchrony (SOA), the interval separating the onsets of the two stimuli.

On the other hand, if the critical variable is the total luminous flux within the first stimulus, then perceptual integration should be relatively unaffected by variation in the ESOA. We found that the degree to which the first and second stimuli are perceptually integrated is, in fact, very largely determined by the ESOA.

We also examined the effect of total luminous flux more directly by introducing a condition in which there was no luminance increment in the first stimulus and another in which the luminance of the first stimulus was incremented for its entire duration. There was no difference in observers' performance between these two conditions.

All experiments except the fourth involved the dot matrix task devised by Hogben and Di Lollo (1974) and employed by Di Lollo and Bourassa (1983). Each display was made up of two brief stimuli presented with a variable interval separating the offset of the first stimulus from the onset of the second (the interstimulus interval, or ISI). The two component stimuli were arrays of 12 dots, positioned on each trial so that they collectively occupied a random selection of 24 of the 25 locations in a $5 \times 5$ matrix. After each presentation, the observer chose which of the matrix locations had been unoccupied.

\section{Method}

Observers. The observers were the first author (C.C.), a colleague familiar with the experimental aims (D.L.), and a paid volunteer naive with regard to the purpose of the research (L.T.). All 3 were highly practiced with the dot matrix task and had normal or corrected-to-normal vision.

The visual display. The $5 \times 5$ dot matrix subtended $4^{\circ}$ of visual angle on a side at the viewing distance of $57 \mathrm{~cm}$. Neighboring dots within a column or row were separated by $1.0^{\circ}$ of visual angle. All displays were generated on the screen of a Hewlett-Packard 1333A oscilloscopic point-plotter equipped with fast P15 phosphor, which decays to $1 \%$ of its initial intensity within $10 \mu \mathrm{sec}$. The stimulus dimensions and display apparatus were identical to those employed by Di Lollo and Bourassa (1983).

The luminance of dots in the first stimulus was first set to a comfortable level and then measured using a calibration procedure similar to that described by Di Lollo (1979). A patch of 81 dots in a $2-\mathrm{mm}$ square was produced, in which individual dots were plotted at the same luminance and refreshed at the same rate as dots in the stimuli themselves (i.e., once every $1 \mathrm{msec}$ ). The luminance of this patch was measured, using a Spectra Spotmeter with $1.0^{\circ}$ luminance probe, which yielded a value of $20.7 \mathrm{~cd} / \mathrm{m}^{2}$ for the standard (unincremented) level of the first stimulus. We then matched the brightness of the 10 -msec second stimulus to that of the 100 -msec unincremented first stimulus (as in Di Lollo \& Bourassa, 1983), using a procedure described by $\mathrm{Di}$ Lollo (1979). This resulted in a luminance of $211 \mathrm{~cd} / \mathrm{m}^{2}$ for the second stimulus. The level of the $20-\mathrm{msec}$ luminance increment was set at $429 \mathrm{~cd} / \mathrm{m}^{2}, 1.3 \mathrm{log}$ units above the standard level, the same increment used by Di Lollo and Bourassa (1983). The viewing surface reflected less than $1 \mathrm{~cd} / \mathrm{m}^{2}$.

Design and Procedure. Observations were carried out in a test cubicle with ambient illumination maintained at low photopic levels. Observers sat in front of the oscilloscope screen, viewing stimuli binocularly with natural pupils from a distance fixed by means of a headrest.

All trials were preceded by presentation of four fixation points positioned at the corners of a square area $8^{\circ}$ on a side. The dot matrix was presented at the center of this area.

Each trial involved a single presentation, which the observer initiated by pressing a button. All displays consisted of a first stimulus with a constant $100-\mathrm{msec}$ duration, an ISI of 0-100 msec, and a second stimulus with a constant 10 -msec duration. In some experimental conditions, a 20 -msec luminance increment occurred $0-80 \mathrm{msec}$ after the onset of the first stimulus.

Following the display, the observer indicated which of the 25 matrix locations appeared to have been unfilled, by entering the row and column coordinates of the location on a set of buttons. These coordinates were then displayed for confirmation as an empty space in an otherwise complete $5 \times 5$ matrix. The observer either 
verified these coordinates or entered a different response. Once a response had been verified, the four fixation points reappeared in readiness for the next trial.

Observers completed 28 - to 10 -min sessions. Each session consisted of 300 trials in one of the seven experimental conditions, 25 at each of six ISIs $(0,20,40,60,80$, and $100 \mathrm{msec})$. The presentation order of ISIs was completely randomized across trials. The 28 sessions were sequenced as four blocks of sessions in which the seven experimental conditions were presented in random order. In total, 100 trials were presented for each ISI in each condition.

Of the seven experimental conditions, one was a no-increment control, in which the same first stimulus was presented without a luminance increment. A second was a continuous-increment control, in which the first stimulus was presented at a constant luminance equal to that of the brief increment in other conditions. The five remaining conditions represented different temporal positions of the 20-msec luminance increment within the first stimulus. The corresponding intervals separating the onset of the first stimulus from that of the luminance increment were $0,20,40,60$, and $80 \mathrm{msec}$.

\section{Results and Discussion}

Figure 1 shows accuracy of performance as a function of ISI for each of the seven conditions, separately for the 3 observers, C.C., L.T., and D.L. Since our interest is in the duration of visible persistence, as measured from offset of the first stimulus, the abscissa in Figure 1 shows ISI, and the ordinate shows the percentage of correct responses. In the upper panel, the parameter chosen to distinguish the five conditions is the duration of the interval extending from the onset of the luminance increment to the offset of the first stimulus: this would be the effective duration (D) of the first stimulus if persistence were reinitiated by the luminance increment. The two constant-luminance conditions are shown, for clarity, in the lower panel.

Several features of the results shown in Figure 1 are worth noting. The first is that the performance curves for each observer form an orderly progression across values of D. As D decreases (that is, as the 20 -msec increment is moved from the beginning of the first stimulus toward its end), the function relating performance to ISI progressively shifts toward longer durations of ISI. In other words, as the presentation of the 20 -msec increment is delayed within the first stimulus, there is a corresponding increase in the ISI that can be tolerated for a given level of performance. In fact, when the curves are compared at the level of $50 \%$ correct performance, it is found that, on the average, a 20 -msec delay of the luminance

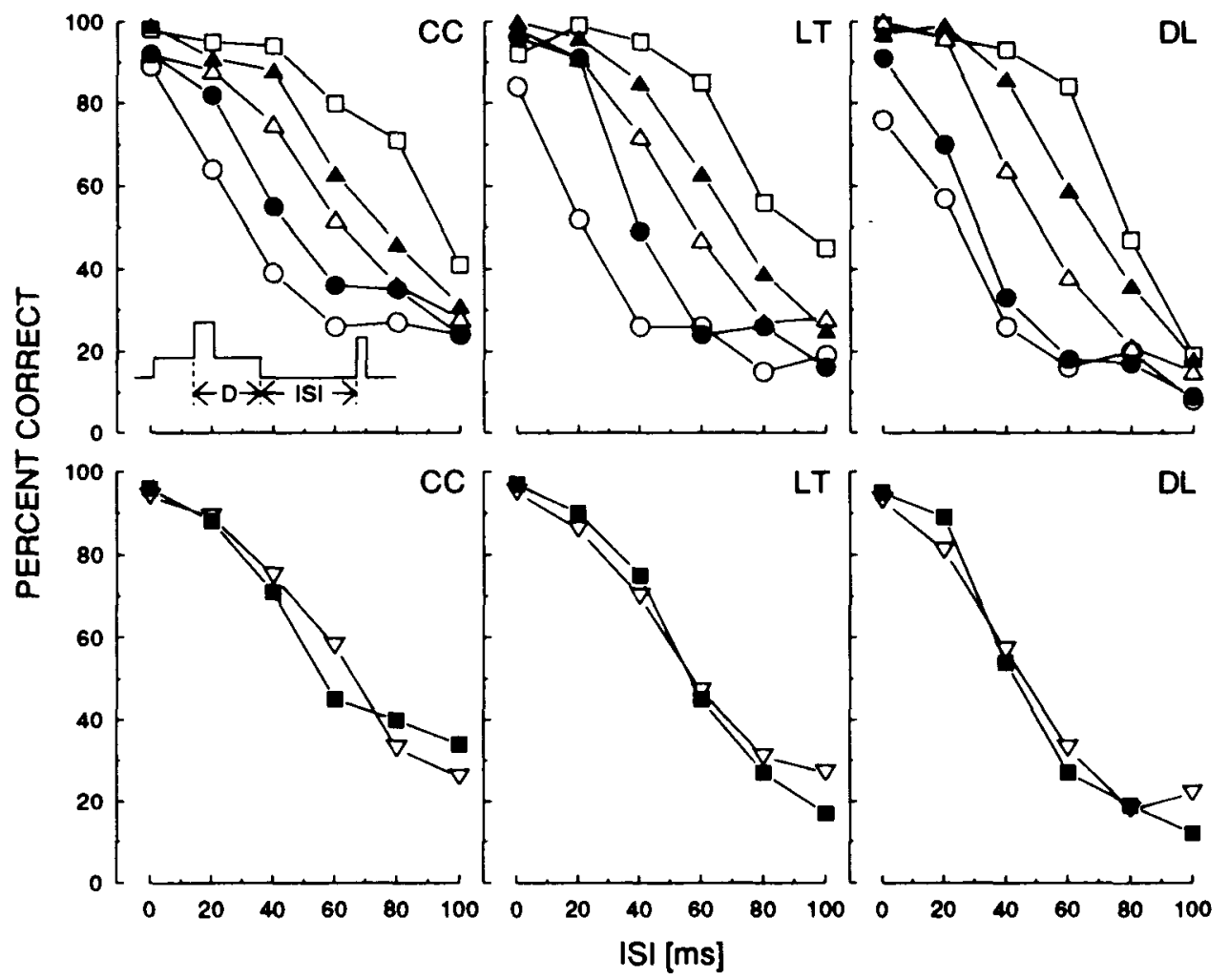

Figure 1. The results of Experiment 1, in which the luminance of a 100 -msec stimulus was incremented for 20 msec at various onset asynchronies. The schematic insert illustrates the effective duration (D) and interstimulus interval (ISI) with reference to the time course of variation in stimulus luminance. Percentage of correct responses is plotted in the upper panels as a function of ISI for five values of D: $20(\square), 40(\Delta)$, $60(\triangle), 80(0)$ and $100 \mathrm{msec}(0)$. The lower panels show results for two control conditions: the $100-\mathrm{msec}$ stimulus presented without an increment $(D)$, and the 100 -msec stimulus presented with luminance at the incremented level for its entire duration $(\nabla)$. Results are for 3 observers (C.C., L.T., and D.L.). 
increment must be paired with a $15-\mathrm{msec}$ increase in the ISI to produce the same level of accuracy. This relationship is shown more clearly in the upper portion of Figure 2.

The second noteworthy feature of the results is that when the increment is presented at the end of the stimulus ( $\mathrm{D}=20 \mathrm{msec}$ ), performance is better than in the noincrement control condition. This replicates the results of Di Lollo and Bourassa (1983). It is clear that this lengthening of persistence is not due to the accompanying increase in total luminous flux within the first stimulus. Indeed, when the luminance increment occurs at the beginning of the first stimulus ( $D=100 \mathrm{msec}$ ), performance is actually reduced relative to the no-increment control conditions, while no difference is observed when the increment is presented at about the middle ( $D=60 \mathrm{msec}$ ) of the stimulus.

A comparison of performance levels in the two noincrement control conditions (illustrated in the lower panel of Figure 1) shows that total luminous flux had no systematic effect on persistence. Despite a $1.3 \mathrm{log}$ unit difference in luminance of the first stimulus in the two conditions, performance levels did not differ for any of the 3 observers.

This general pattern of results is entirely consonant with Di Lollo and Bourassa's (1983) proposition that the lu-

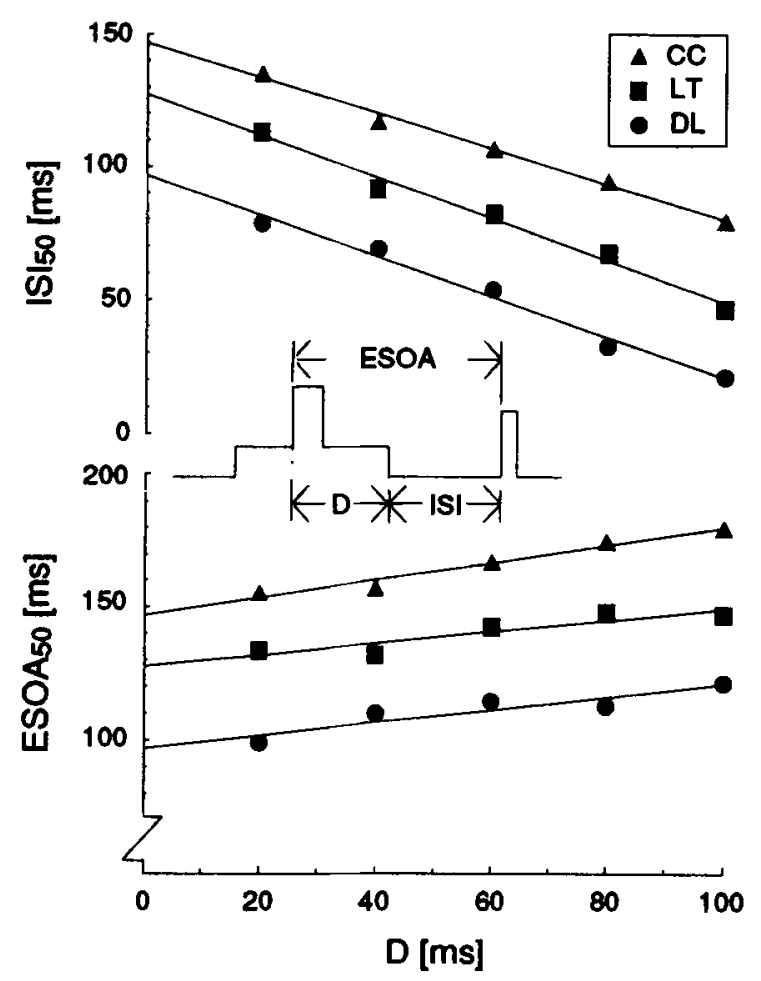

Figure 2. The results of Experiment 1. The interstimulus interval (ISI) and equivalent effective stimulus onset asynchrony (ESOA) that produced $50 \%$ accuracy on the matrix integration task are plotted as a function of the effective duration (D) for 3 observers (C.C., L.T., D.L.). The fitted curves are least squares linear regression solutions. minance increment acts to initiate a new period of activity in the persistence mechanism. As D increases-that is, as the increment moves from the end toward the beginning of the first stimulus-a systematic decrease in performance occurs at all ISIs. This would be expected if the termination of persistence was time-locked to the onset of the luminance increment.

In order to examine the temporal relationship between persistence and the luminance increment in greater detail, we selected an arbitrary criterion level of $50 \%$ accuracy and derived point estimates of the corresponding ISIs (denoted as ISI $_{50}$ ) by fitting cumulative normal curves to the data illustrated in Figure 1. These ISI $_{50}$ S are plotted as a function of $\mathrm{D}$ in the upper portion of Figure 2. The corresponding ESOA $\mathrm{E}_{50} \mathrm{~s}-$ the sum of the $\mathrm{ISI}_{50} \mathrm{~S}$ and their paired D values-are plotted in the lower portion of this figure. The relationship between these parameters and D is closely approximated by the least squares linear regression curves shown fitted to the data in Figure 2. In the case of ESOA $_{50}$, these curves account for $83 \%-97 \%$ of the variance for the 3 observers.

If the activity initiated by the luminance increment had a constant duration and a constant latency of initiation, then the extent of overlap between persistence of the first stimulus and visibility of the second stimulus (and hence, response accuracy) would depend only on the time between onset of the increment and onset of the second stimulus-that is, on the ESOA. Figure 2 shows that this is approximately the case: ESOA $\mathbf{s o}_{\mathbf{5}}$ varies much less than ISI $_{30}$ as a function of the temporal position of the luminance increment within the first stimulus. In the upper portion of this figure, $20-\mathrm{msec}$ increments in $\mathrm{D}$ are seen to lead to approximately $15-\mathrm{msec}$ decrements in ISI $_{50}$. By contrast, the lower portion of Figure 2 shows that the same increment in $D$ produces, on the average, only about a 5-msec change in ESOA $_{50}$. The implication is that, as $D$ varies, constant performance would be more nearly attained by holding the ESOA constant than by holding the ISI constant.

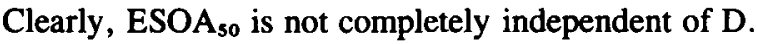
The positive gradients of the regression curves fitted to ESOA $_{50} \mathrm{~s}$ in the lower portion of Figure 2 differ significantly from zero for all 3 observers. The ESOA required to produce the criterion level of performance, then, was not constant, but rather increased by a total of $21 \mathrm{msec}$ on the average as the luminance increment was moved from the end to the beginning of the first stimulus. It follows that if the luminance increment did initiate a new period of activity in the persistence mechanism, then the duration of this activity, and/or its latency of initiation, varied as a function of the temporal position of the luminance increment within the first stimulus.

In clarifying the functional properties of the persistence mechanism, it is of considerable interest to know whether the luminance increment completely reinitiated activity in the persistence mechanism, or whether it instead modulated activity that had already been initiated by the preceding stimulus segment. In the latter case, we might expect 
the observed variation in $\mathrm{ESOA}_{50}$ to arise, in part, from variation in the duration of the stimulus segment preceding the luminance increment. Experiment 2 was designed to investigate this possibility.

\section{EXPERIMENT 2}

The preceding experiment showed that performance was predominantly determined by the interval between the onset of the luminance increment and the onset of the second stimulus (the ESOA) rather than by the ISI, the interval between the termination of the first stimulus and the onset of the second. However, it was also clear that the ESOA did not fully account for performance. A possible explanation for the observed variation in the ESOA required for constant performance is suggested by the finding of Di Lollo and Bourassa (1983, Experiment 4) that the duration of persistence varies continuously as a function of the magnitude of the luminance increment. At least two variables that might similarly have modified the magnitude of the response to the luminance increment were confounded with D in Experiment 1 of the present sturdy. First, variation in D entailed variation in the temporal separation of stimulus onset and the onset of the luminance increment and, therefore, in the likely extent of temporal interactions between the visual responses to these stimulus events. Second, variation in D might have resulted in variation in the degree of local light adaptation that preceded the luminance increment and, therefore, in the magnitude of the luminance increment relative to the prevailing adaptation level.

It is conceivable, then, that the variation in $\mathrm{ESOA}_{50}$ that occurred as a function of $\mathrm{D}$ was attributable to variation in the duration of the stimulus segment preceding the luminance increment. On the other hand, it is possible that the magnitude of the luminance increment employed in Experiment 1 was high enough for this initial stimulus segment to have had a negligible effect on the response of the persistence mechanism, and that the first stimulus effectively commenced with the onset of the luminance increment. In the latter case, it would be necessary to look at other factors to explain the variation in $\mathrm{ESOA}_{30}$ as a function of $D$.

In Experiment 2, we tested whether the initial segment of the first stimulus had any effect on the visible persistence generated by the luminance increment, by repeating Experiment 1 and simply removing the initial segment.

\section{Method}

Experiment 2 replicated Experiment 1 in all respects, save that the segment of the first stimulus that preceded the luminance increment in that experiment was omitted. Only the conditions that corresponded to the five levels of $D$ in Experiment 1 were examined. In this case, D was also the physical duration of the first stimulus.

\section{Results and Discussion}

The ESOA ${ }_{50}$ values estimated in Experiment 2, together with the corresponding values obtained in Experiment 1, are plotted in Figure 3. Removing the segment that pre-

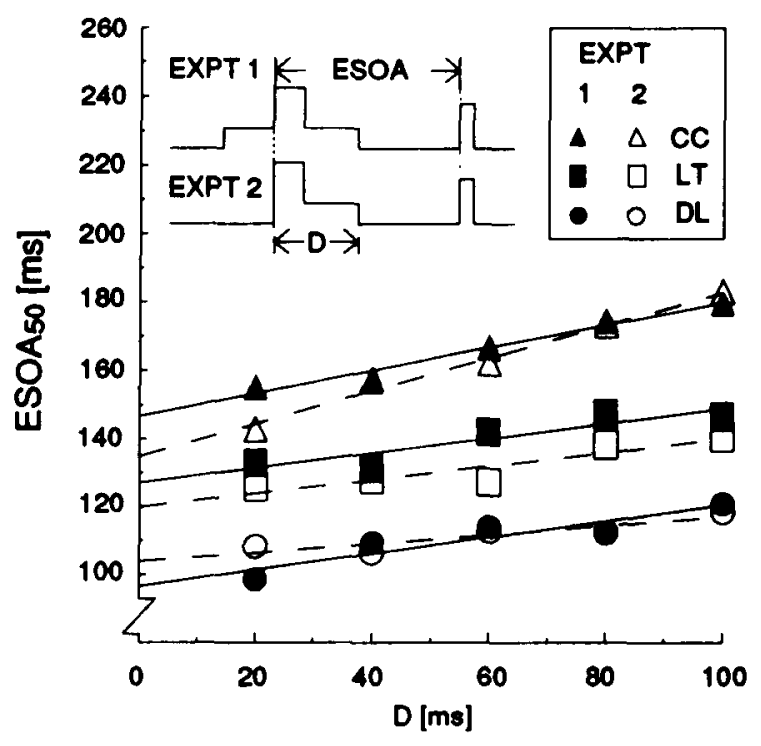

Figure 3. The results of Experiment 2, in which the segment of the first stimulus that had preceded the luminance increment in Experiment 1 was omitted. The effective stimulus onset asynchrony (ESOA) that produced $50 \%$ accuracy on the matrix integration task is plotted as a function of the effective duration (D) for 3 observers (C.C., L.T., D.L.), together with the corresponding ESOA estimates from Experiment 1. Least squares linear regression curves are shown. For clarity, the curves for Observers L.T. and C.C. have been displaced upward by 20 and $\mathbf{4 0} \mathrm{msec}$, respectively.

ceded the luminance increment in the first stimulus had no detectable effect on performance for 2 of the $3 \mathrm{ob}$ servers. The 3rd observer (L.T.) did consistently produce slightly lower ESOA so values (i.e., a shorter persistence) when this initial segment of the stimulus was removed. The difference was not statistically significant, however. Within the limits of experimental error, then, the ESOA required to produce a criterion level of accuracy was found to vary directly as a function of $D$ in exactly the same manner as in Experiment 1.

It is apparent from these results that the variation in ESOA $_{\text {so }}$ observed as a function of $\mathrm{D}$ is not attributable to any effect associated with the stimulus segment that precedes the luminance increment. In fact, it is clear that this variation must be associated with change in the duration of the stimulus segment that follows this increment. Figure 3 shows that each $20-\mathrm{msec}$ increase in the duration of this following segment increased persistence by approximately $5 \mathrm{msec}$.

The fact that removing the initial stimulus segment had no substantial effect on persistence provides further confirmation that, when this segment is present, activity in the persistence mechanism is time-locked to onset of the luminance increment and not to the initial stimulus onset. We can be confident, then, that the systematic change in ESOA $\mathrm{A}_{\text {so }}$ that accompanies change in D indicates change in the duration of activity that the luminance increment initiates in the persistence mechanism, and/or in the latency with which this activity is initiated. It seems un- 
likely that the duration of activity initiated by the luminance increment was independent of $D$. Had this been true, the fact that $\mathrm{ESOA}_{50}$ varied directly rather than inversely with stimulus duration would require the latency of initiation of this activity to have been longer for stimuli of greater energy. In general, latencies of visual responses show precisely the opposite effect, decreasing asymptotically as stimulus energy increases (Arden \& Weale, 1954; Roufs, 1963). The more plausible interpretation, then, is that increasing the duration of the stimulus segment that follows the luminance increment increases the duration of the activity initiated by this increment, perhaps by sustaining activity at higher levels prior to stimulus offset.

\section{EXPERIMENT 3}

Comparison of the ESOA so $_{50}$ estimates obtained in Experiments 1 and 2 shows that the duration of the stimulus segment preceding the luminance increment had essentially no effect on the duration of persistence. The same relationship of performance to $D$ was found in the two experiments, despite the fact that, in one case, the stimulus segment preceding the luminance increment was entirely absent and, in the other, it was up to $80 \mathrm{msec}$ in duration. In order to confirm more directly that the initial stimulus segment has no effect on persistence (and, conversely, that the previously observed variation in persistence was attributable to change in the stimulus segment following the increment), we conducted a third experiment in which we varied the duration of this initial segment while holding the duration of $\mathrm{D}$ constant. This experiment, in which we simply removed the stimulus segment that had followed the luminance increment in Experiment 1, complements Experiment 2, in which we removed the segment preceding the luminance increment. The results were as predicted, given the results of the preceding experiments.

\section{Method}

Here again we replicated Experiment 1, save that the segment of the first stimulus that followed the luminance increment was omitted. Only the conditions that corresponded to the five levels of $D$ in Experiment 1 were examined. In this case, $D$ itself remained constant at $20 \mathrm{msec}$ (the duration of the luminance increment), while the duration of the initial segment of the first stimulus varied from 0 to $80 \mathrm{msec}$.

\section{Results and Discussion}

Figure 4 shows ESOA $_{s o}$ as a function of the total duration of the first stimulus, together with least-squares linear regression curves fitted to the data for each of the $3 \mathrm{ob}$ servers. In no case did the gradient of a curve differ significantly from zero. The duration of persistence, then, is essentially independent of the duration of the initial segment of the first stimulus.

It must be noted that the results of this experiment are dependent on the magnitude of the luminance increment. In the present case, in which a large luminance increment was employed, varying the duration of the stimulus seg-

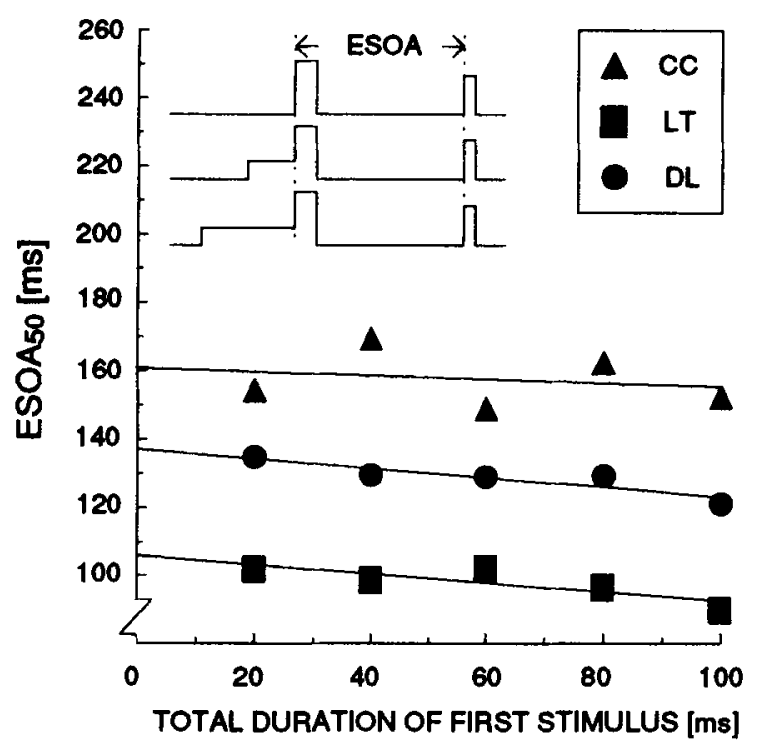

Figure 4. The results of Experiment 3, in which the segment that had followed the luminance increment in the first stimulus (in Experiment 1) was omitted. The effective stimulus onset asynchrony (ESOA) that produced $50 \%$ response accuracy on the matrix integration task is plotted as a function of total duration (i.e., the duration of the initial dimmer segment of the first stimulus plus the constant 20-msec duration of the luminance increment). Least squares linear regression curves are shown. For clarity, data for Observers D.L. and C.C. have been displaced upward by 20 and $\mathbf{4 0} \mathrm{msec}$, respectively.

ment preceding the luminance increment had no significant effect on performance. Had a zero increment been used (thereby reducing the stimulus to a rectangular pulse of varying duration), the ESOA $A_{\text {so }}$ measured from the merely notional onset of the luminance increment (equivalent to ISI $_{50}$ plus a constant $20 \mathrm{msec}$ ) would have decreased as a function of the total stimulus duration. This result is directly implied by the inverse duration effect, previously noted to characterize visible persistence (Coltheart, 1980). The data of Di Lollo and Bourassa (1983) suggest that, at intermediate magnitudes of the luminance increment, we could expect intermediate gradients of the curve relating ESOA $_{\mathbf{s o}}$ to stimulus duration. In their third experiment, Di Lollo and Bourassa demonstrated that increasing the magnitude of the luminance increment had little effect on performance when the duration of the preceding stimulus segment was zero (corresponding to the 20msec stimulus duration in the present experiment). In their second experiment, they showed that similar increases produced a progressive improvement in performance when the stimulus segment preceding the luminance increment had a duration of $80 \mathrm{msec}$. Taken together, these two results suggest that a progressive increase in the magnitude of a luminance increment placed at the end of a stimulus is matched by a progressive increase in the gradient of the function relating ESOA $_{50}$ to stimulus duration.

The present results demonstrate that when a luminance increment of sufficient magnitude is used, the initial 
(dimmer) segment of the stimulus has no significant effect on persistence. As far as the persistence mechanism is concerned, stimulus onset occurs at the onset of the luminance increment.

This result would be somewhat trivial if the increment, being 1.3 log units higher in luminance, had in some way prevented the preceding stimulus segment from being visible (i.e., from initiating activity in the persistence mechanism). In Experiments 4 and 5, we demonstrated that the stimulus segment preceding the luminance increment is not masked in this sense; that it remains visible and can contribute to temporal integration.

\section{EXPERIMENT 4}

The stimuli in Experiment 3 were made up of an initial dimmer segment of variable duration, followed by a 20 -msec $1.3 \mathrm{log}$ unit luminance increment. If the initial segment preceding the luminance increment were simply masked because of the overwhelming magnitude of the luminance increment, we would not expect this segment to have any influence on simple perceptual judgments such as simultaneity. For example, an observer asked to synchronize a temporal marker with such a stimulus would align it with reference to the increment alone, rather than with reference to the increment plus the supposedly masked initial segment.

In Experiment 4, we asked observers to perform precisely this task of aligning a 10 -msec temporal marker with the temporal midpoint of the stimuli used in Experiment 3.

\section{Method}

The display employed in Experiment 4 resembled the dot matrix display used in the preceding experiments. Again it consisted of the asynchronous presentation of two stimuli, each of which was made up of dots occupying a subset of locations in the same $5 \times 5$ dot matrix. On any trial, dots in one of these stimuli filled the 10 locations in the two left-most columns of the matrix. Dots in the other stimulus filled the corresponding locations in the two rightmost columns (the left-right assignment of the two stimuli being randomized across trials). The middle column of the matrix contained only a single fixation point, which was positioned at the center of the display and present throughout each session.

Dots in one stimulus were illuminated identically to those constituting the briefly incremented stimuli used in Experiment 3. Presentation of this stimulus terminated with a 20 -msec luminance increment and had a total duration that varied across experimental conditions from 20 to $100 \mathrm{msec}$. The other stimulus, the temporal marker, was presented for a constant $10 \mathrm{msec}$ at a brightness matched to the average brightness of the longer stimulus.

When two stimuli are presented in close spatial proximity with some asynchrony, apparent motion is often perceived, as though a single stimulus had moved from the location of the first stimulus to that of the second (see, e.g., Kolers, 1972). When this effect is undesirable, as, for example, when observers are trying to match the brightness of two stimuli of unequal duration, it can easily be minimized through presentation of the two stimuli so that their temporal midpoints are aligned (Di Lollo \& Finley, 1986). We made use of this fact in Experiment 4, by employing exactly the reverse procedure: observers were asked to minimize the apparent motion between the two stimuli, so that the brief marker stimulus would be aligned with the temporal midpoint of the other, longer, stimu- lus. This technique was chosen because apparent motion is by far the most conspicuous cue to temporal order in these circumstances, and judgments of the direction of apparent motion proved to be a more feasible task than requiring an observer simultaneously to judge and to attempt to align the temporal midpoints of the two stimuli.

On each trial, the observer indicated the direction of apparent motion generated by the asynchronous presentation of the two stimuli. Over successive trials, the onset asynchrony of these stimuli was adjusted with the standard PEST procedure (Taylor \& Creelman, 1967), to yield an estimate of the asynchrony at which minimum apparent motion was perceived (that is, the asynchrony at which the direction of apparent motion was as likely to be toward as away from the briefer stimulus).

In each session, observers were presented with one of five durations of the longer stimulus $(20,40,60,80$, and $100 \mathrm{msec})$, and three estimates of the asynchrony producing minimum apparent motion were obtained. The median of these three estimates was taken as a session estimate. Two observers (C.C. and L.T.) completed 15 sessions, in which the five durations were presented in random order in three consecutive blocks of sessions.

\section{Results and Discussion}

Figure 5 shows the onset asynchrony that produced minimum apparent motion plotted as a function of the total duration of the longer (briefly incremented) stimulus for each observer. Each point is the mean of estimates produced in three sessions.

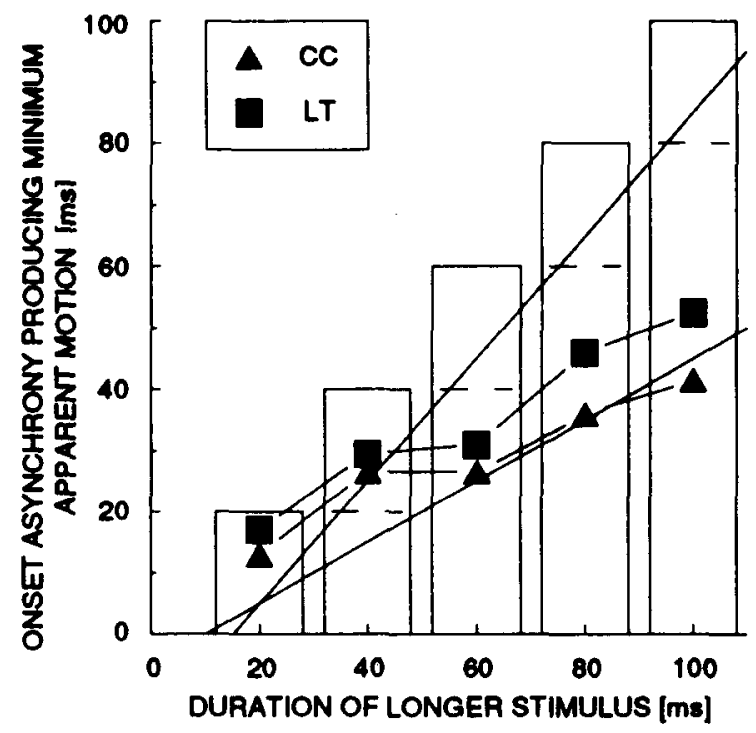

Figure 5. The results of Experiment 4, in which Observers C.C. and L.T. adjusted the onset asynchrony of two adjacent stimuli (groups of dots) until apparent motion between these stimuli was equally likely to be judged either "away from" or "toward" the briefer stimulus of constant 10-msec duration. This onset asynchrony is plotted here as a function of the duration of the longer stimulus for 2 observers. Dots in the longer stimuli were illuminated identically to those constituting the first stimulus in Experiment 3; that is, they were of varying duration and terminated with a 20-msec luminance increment. These stimuli are represented schematically by the vertical bars, with the 20-msec segment above the dashed line on each bar representing the terminal luminance increment. See text for an explanation of the lines illustrated. 
The briefly incremented stimuli are represented schematically by vertical bars in Figure 5, with the 20 -msec portion above the dashed line on each bar representing the terminal luminance increment. The lower of the two straight lines drawn in this figure marks the onset asynchrony required for the physical temporal midpoints of the two stimuli to be coincident. The upper of these two lines marks the asynchrony required for the temporal midpoint of the briefer stimulus to be coincident with that of the brief luminance increment at the end of the longer stimulus.

It is not to be expected that the empirical curves will lie along either of the straight lines drawn in Figure 5. The fact that such stimuli have persistence-that is, that they are visible for durations that exceed their physical durations-implies that the temporal midpoint of the period of visibility of a stimulus will not coincide with its physical midpoint. Nevertheless, we know that persistence is constant across experimental conditions for both the longer stimulus (as demonstrated in Experiment 3) and the briefer stimulus (which is invariant across conditions). Under such conditions, if we could adjust the position of the two lines drawn in Figure 5 so that they represented coincident midpoints of periods of visibility rather than the coincident physical midpoints of the two stimuli, then only the ordinate intercepts of these lines would change. Their gradients would remain the same. The ordinate intercept is suspect in any event, given possible differences in the latencies with which the two stimuli initiate critical visual responses.

If the segment of the longer stimulus that preceded the luminance increment was not visible to the observerthat is, if only the brief luminance increment was seenthen increasing the duration of this segment should have had no effect on the asynchrony at which minimum apparent motion was observed. In other words, the curves shown in Figure 5 should have been parallel to the upper of the two straight lines. Instead, the gradient of these curves can be seen to approximate that of the lower line. Observers positioned the briefer stimulus about midway between the onset and offset of the longer stimulus.

These results provide empirical support for the strong subjective impression of the observers that the segment of the longer stimulus that preceded the luminance increment was clearly visible. In Experiment 5, we demonstrated that this stimulus segment was also available to the pattern integration mechanism.

\section{EXPERIMENT 5}

A further means of demonstrating that the brief luminance increment presented during long-lasting displays did not completely mask the preceding stimulus segment in Experiments 1 and 3 is to show that the presence of this segment can facilitate pattern integration when the temporal order of the briefly incremented long-lasting stimulus and the brief second stimulus is reversed. If this initial segment of the longer (now second) display is visible, then increasing its duration while holding the ESOA (the temporal separation of the onset of the first stimulus and the onset of the luminance increment in the second stimulus) constant should increase the length of time for which the two stimuli appear to be simultaneously present and, therefore, should improve performance. By the same token, an increase in the duration of this segment should increase the ESOA at which a selected performance level is attained.

The persistence of these briefly incremented stimuli was previously shown to be independent of the duration of the stimulus segment that preceded the luminance increment (Experiment 3). Hence, if this segment is not visible (in the sense of being perceptually unavailable to the processes responsible for pattern integration), then varying its duration should have no effect on the duration of visibility of this stimulus and, consequently, no effect on task performance.

We show here that task performance does, in fact, improve as the duration of the initial segment of the second stimulus increases.

\section{Method}

The method and procedure were as in Experiment 3, save that the first and second stimuli in that experiment were presented here in reverse order. The first stimulus had a $10-$ msec duration and constant intensity; it was followed by an ISI of $0,20,40,60,80$, or $100 \mathrm{msec}$. The second stimulus had a $20-$ to $100-\mathrm{msec}$ total duration and terminated with a $20-\mathrm{msec}$ luminance increment.

\section{Results and Discussion}

Figure 6 illustrates the ESOA $_{50}$ estimates obtained for each observer for each of the five durations of the segment that preceded the luminance increment in the second stimulus. Curves for all 3 observers show a positive trend. Hence, at a given ESOA, performance improved as the duration of the initial segment of the second stimulus was increased.

The simplest interpretation of these results is that moving the onset of the second stimulus toward the offset of the first stimulus facilitated temporal integration by increasing the length of time for which the two stimuli were seen simultaneously. However, it might be argued that increasing the duration of the initial segment of the second stimulus did not improve temporal integration, but that the resulting increase in $\mathrm{ESOA}_{50}$ was entirely due to the accompanying reduction in ISI at a given ESOA. Which interpretation is more accurate is not of concern here, in that both presuppose that the initial stimulus segment was visible - the point that this experiment is intended to demonstrate.

In Experiment 3, the initial segment of the briefly incremented stimulus was shown to have no appreciable effect on the visibility of the remainder of this stimulus. It follows that, had this initial segment not itself been visible, varying its duration should not have had any effect on task performance. There can be little doubt, then, that 


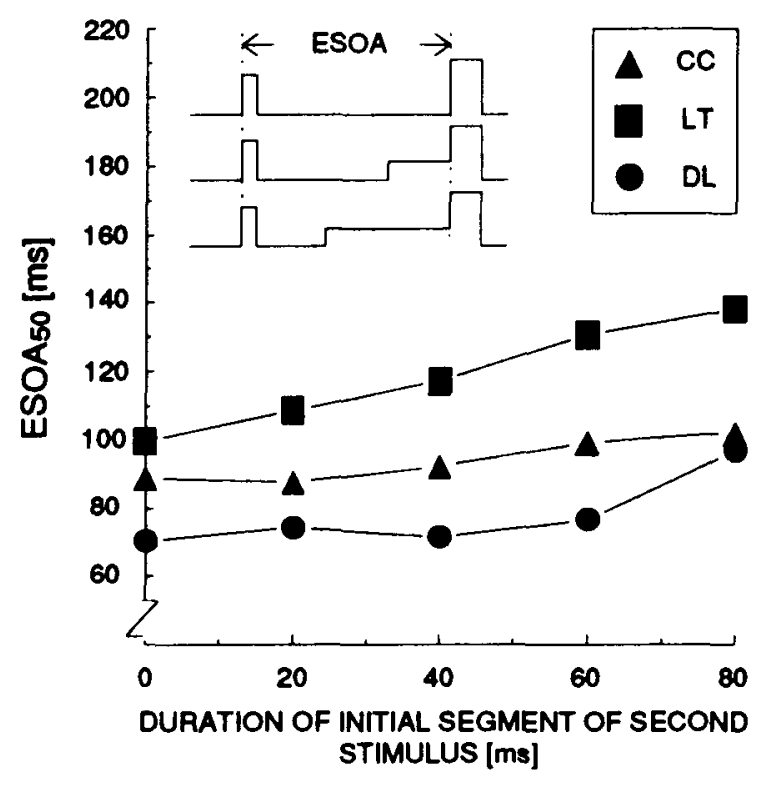

Figure 6. The results of Experiment 5, in which the temporal order of the stimuli used in Experiment 3 was reversed; that is, the first stimulus consisted of a simple rectangular pulse of constant duration, and the second of a 20 -msec luminance increment preceded by a dimmer segment of varying duration. The effective stimulus onset asynchrony (ESOA) that produced 50\% accuracy on the matrix integration task is plotted as a function of the duration of the initial segment of the second stimulus. For clarity, data for Observer L.T. have been displaced upward by 20 msec.

the variation in stimulus visibility evidenced by change in $\mathrm{ESOA}_{30}$ in the present experiment was due to variation in the visibility of the initial stimulus segment itself.

\section{GENERAL DISCUSSION}

The results of Experiment 1 show that the improved temporal integration that Di Lollo and Bourassa (1983) obtained by presenting a brief luminance increment at the end of a long-lasting first stimulus was not an artifact of the difference in total luminous flux between the incremented and unincremented first stimuli. We found no difference between the two constant-luminance conditions, even though one stimulus was 20 times the luminance of the other. The absence of an effect of stimulus luminance, together with the similar result that $\mathrm{Di}$ Lollo and Bourassa (1983) obtained with briefer $(20-\mathrm{msec})$ stimuli, is clearly at odds with the direct effect of stimulus luminance that would be expected if other persistence phenomena, such as retinal afterimages, had mediated performance-although it does cast doubt on the generality of the inverse intensity effect (Coltheart, 1980).

We also confirmed that the termination of persistence is time-locked to presentation of the brief luminance increment rather than to stimulus onset, by showing, in Experiment 1 , that ESOA $_{\mathbf{s o}}$ covaries in a near-linear fashion with the temporal position of the luminance increment within the long-lasting first stimulus and, in Experiment 2, that removing the stimulus segment preceding the luminance increment has no effect on performance.

Experiments 4 and 5 have demonstrated that, despite the fact that the luminance increment had a magnitude sufficient to achieve complete reinitiation of persistence, the stimulus segment preceding the luminance increment remained visible and available to the processes responsible for the temporal integration of pattern. These findings undermine any argument to the effect that the initial stimulus segment made no contribution to the duration of persistence because it was "masked."

Taken together, these results demonstrate that the luminance increment did not merely supplement on-going activity initiated at stimulus onset, but acted to reinitiate activity in the persistence mechanism.

In the discussion that follows, we will reevaluate the two hypotheses regarding the nature of the mechanism of visible persistence considered by Di Lollo and Bourassa (1983). We will then show that the present experimental findings can be accommodated within a simple explanatory framework, based on a single-process feedbackcontrolled mechanism.

The processing hypothesis put forward by Di Lollo and Bourassa (1983) holds that the duration of the activity that a stimulus initiates in the persistence mechanism corresponds to the duration of very early stages in a chain of processing events triggered by a change in visual stimulation. Once initiated, whether by the onset of a new stimulus or by an increment in the luminance of an existing stimulus, this activity is held to continue for a period that reflects the time course of these initial encoding processes. Hence, the processing hypothesis is able to account for the inferred independence of the duration of activity in the persistence mechanism from the duration of the inducing stimulus (Di Lollo, 1980; Di Lollo \& Bourassa, 1983).

According to the processing hypothesis, the duration of the activity initiated in the persistence mechanism varies as a function of the parameters of the initiating stimulus and the degree to which previously processed stimulus attributes (such as shape, spatial location, orientation, and brightness) remain invariant during the change in stimulation that triggers this activity. For example, Di Lollo and Bourassa (1983) argue that a luminance increment in an existing stimulus changes only a subset of stimulus attributes, thereby producing lower processing demands than a completely new stimulus and, consequently, a shorter period of activity in the mechanism underlying persistence.

The processing hypothesis does not sit comfortably with the results of Experiment 3, in which we varied the duration of the stimulus segment preceding the luminance increment over a range of $80 \mathrm{msec}$ without producing any significant change in the duration of persistence. Experiments 4 and 5 demonstrated that at least some of the perceptual attributes of this initial stimulus segment were encoded. Hence, the processing hypothesis would have predicted an inverse relationship between the duration of 
persistence and the duration of this initial stimulus segment rather than the independence of these variables actually observed. Indeed, in general terms, the processing hypothesis would predict an exact tradeoff between the duration of visible persistence and the duration of the stimulus-that is, a function with a slope of -1.0 , reflecting a constant interval between stimulus onset and the end of persistence. Repeated observations over a wide range of conditions by Hogben and Di Lollo (1990) estimate this slope at -0.4 instead, forcing a reevaluation or abandonment of the processing hypothesis.

Processes of a different kind are central to the on/offresponse hypothesis proposed initially by Serviere et al. (1977a, 1977b) and elaborated by Coltheart (1980). According to this hypothesis, the onset of the activity underlying persistence is time-locked to the first positive peak of the cortical on response elicited by stimulus onset, and the termination of this activity is similarly time-locked to the cortical off response elicited by stimulus offset. This hypothesis originates in electrophysiological studies, which showed the latency of the cortical off response measured from stimulus onset to reach a minimum, asymptotic value as the duration of the inducing stimulus decreased toward approximately $60 \mathrm{msec}$ (Serviere et al., 1977b). The supposed link between the latency of the off response and termination of persistence readily accounts for the inverse relationship between stimulus duration and the duration of persistence.

It would be difficult to evaluate this hypothesis with reference to the present experimental task, without a more detailed knowledge of the way in which the multiple luminance transients and accompanying variations in visual adaptation levels might interact in a briefly incremented display to determine the latencies of the cortical on and off responses. Without adequate theoretical constraints of this kind, a seemingly plausible explanation based on this general hypothesis could be offered for virtually any pattern of results that might have been obtained. For example, this hypothesis can account for Di Lollo and Bourassa's (1983) finding that a luminance increment presented at the end of a long-lasting display has a shorter duration of persistence than a completely new stimulus of the same duration, simply by proposing a difference in the latency with which the offset of the luminance increment and the offset of the stimulus of matching duration elicited the cortical off response.

The on/off response hypothesis does not incorporate sufficient theoretical constraints on the behavior of the persistence mechanism to allow confident predictions to be made concerning experimental outcomes.

A single-process inhibitory feedback model. In the remainder of this paper, we will put forward a somewhat different conceptualization of the persistence mechanism. This derives its broad outline from assumptions that are often implicit in the literature in this area, and its detail from our attempts to produce a parsimonious account of the present findings. Our emphasis here is on present- ing a heuristic conceptual framework for investigations in this area.

We propose that a single process is largely responsible for persistence in the pattern integration task. This process is held to display a continuously variable level of activity that responds to change in stimulus luminance throughout the time course of stimulation. A stimulus remains visible as long as the activity that it initiates remains above a threshold level.

This process is envisaged as a simple feedback control system in which the time course of variation in system activity, or output, is determined by the time course of variation in the level of sensory input in conjunction with the temporal characteristics of the excitatory and inhibitory feedback components of the system response. In the conceptual framework outlined here, system input is broadly equated with stimulus luminance, although the neural response encoding luminance may be modulated by processes that precede input to the persistence mechanism. System output is equated with the strength of activity in the persistence mechanism.

Clearly, any attempt to provide a comprehensive account of a persistence mechanism of this kind must ultimately take into account the spatial dimension of stimulation and the spatial integration properties of the mechanism. These factors, which are of particular importance in relation to the persistence of moving stimuli, are addressed elsewhere (Clark \& Hogben, 1990).

The precise specification of the required temporal characteristics of the excitatory and inhibitory feedback components of the system response is a matter for more systematic evaluation; it requires a more complex mathematical description than we intend to present here. However, the manner in which a system of this kind might function to produce the results obtained in the present study can readily be illustrated with a crude model of persistence incorporating a simple approximation to the time response of the system.

The proposed system incorporates two features characteristic of biological systems: an asymptotic, maximum output level, and adaptation in the form of an inhibitory feedback mechanism, which causes the level of output to decrease over time even at the highest levels of sustained input. We assume that the rate of decrease in system output will have its minimum value at any sustained input (luminance) level sufficient to produce an initial maximum output, and its maximum value at the zero input level that follows stimulus offset.

In general, the rate of change in system output could be expected to decrease as the output level approaches the equilibrium level, which would ultimately be attained with sustained input--that is, as the difference between the current and equilibrium output level diminishes (Baker, 1963; Crawford, 1947). However, for the purpose of modeling the system, we will assume that the decrease in system output is linear for up to $100 \mathrm{msec}$ following a change in stimulus luminance (the range of stimulus durations 
used in this study). Evidence that activity in the persistence mechanism continues for very much longer than $100 \mathrm{msec}$ (see, e.g., Lovegrove et al., 1986) suggests that equilibrium output levels are not approached within this period. Hence, the assumption of linearity may well provide a good approximation to the actual system response.

To demonstrate how this system might operate to produce the results obtained in the present study, we will first consider the response of the system to a simple rectangular pulse that is of sufficient luminance to raise activity to near asymptotic levels and to hold the subsequent rate of decrease in output to its minimum level. This situation is illustrated in Figure 7, which, for purposes of clarity, ignores the system response latency in showing the hypothetical time response of the system mapped directly to the time course of stimulation.

The upper portion of Figure 7 shows the time course of variation in system output corresponding to the various patterns of system input illustrated in the lower portion of the figure. The left panel depicts the maximum system response produced by both of the two control stimuli in Experiment 1. Output rises rapidly to a nearmaximum level immediately following stimulus onset, declines at a constant minimum rate while input is sustained above some level, and then falls at a maximum rate once the stimulus is turned off (eventually falling below the threshold of visibility represented here by the horizontal axis). The duration of persistence is that of the interval bounded by the two dashed vertical lines in the left panel: the time required for output to fall below the threshold level once the stimulus has been turned off. The right panel illustrates how transient masking of the segment that followed the luminance increment in the first stimulus (in Experiment 1; represented by the dotted segments of the system input functions) might account for the results, given the input-output relationships proposed. A temporary drop in effective stimulus luminance to levels below those required to sustain the minimum rate of decrease in system output will produce a shorter duration of persistence than that which is attained when the minimum rate of decrease is sustained until stimulus offset (compare curve A with the maximum system response illustrated in both panels). This effect is progressively countered (e.g., curve B) and reversed (e.g., curve C) by delaying the luminance increment within the $100-\mathrm{msec}$ stimulus (thereby returning system output to nearmaximum levels with less time to decrease before stimulus offset occurs).

This model immediately explains why the large difference in luminance between stimuli in the continuousluminance-increment and no-increment conditions of Experiment 1 had no effect on performance: specifically, that both the unincremented and incremented luminance levels were sufficient to elicit the maximum system response with the minimum response latency. Provided that a stimulus is capable of eliciting this response (illustrated in the left panel of Figure 7), stimulus intensity will have no effect on persistence, save insofar as it might affect the latency with which the initial peak output level is achieved. It is conceivable that dimmer stimuli would elicit this peak response with a longer latency and therefore produce longer persistence, offering an explanation for the inverse intensity effect (Coltheart, 1980).

This model also accounts for the approximately linear relationship between the duration of the inducing stimulus and duration of persistence that we have repeatedly found in this laboratory for simple rectangular pulses and that was obtained in Experiment 2 for inducing stimuli commencing with a 20 -msec luminance increment. For stimuli of up to at least $100-\mathrm{msec}$ duration and for a similar period following stimulus offset, the level of system output (activity) predicted by the model can be represented as a function of time by the piecewise equation $[f(t)=$ $-m_{1} t+c_{1},(t \leq D) ; f(t)=-m_{2}(t-D)+f(D)$, $(t>D)]$, where $t$ is time from stimulus onset and $D$ the duration of the inducing stimulus. The constants $m_{1}$ and $m_{2}$ are the rates of decrease in system output during and after stimulus presentation, respectively, and $c_{1}$ the initial peak level of system output that immediately follows stimulus onset. The persistence of a stimulus $(P)$ is the time required for output to fall from the level attained at stimulus offset [i.e., $f(D)$ ] to some threshold level $\left(A^{\prime}\right)$ at the rate prevailing after stimulus offset (i.e., $m_{2}$ ). This relationship can be represented by the equation $P=\left[f(D)-A^{\prime}\right] / \boldsymbol{m}_{\mathbf{2}}$, which, by expansion, yields the equation

$$
P=\frac{-m_{1}}{m_{2}} \mathrm{D}+\frac{c_{1}-A^{\prime}}{m_{2}}
$$

The terms $\left(A^{\prime}-c_{1}\right) / m_{2}$ and $-m_{1} / m_{2}$ in Equation 1 are zonstants, the latter being invariably negative under

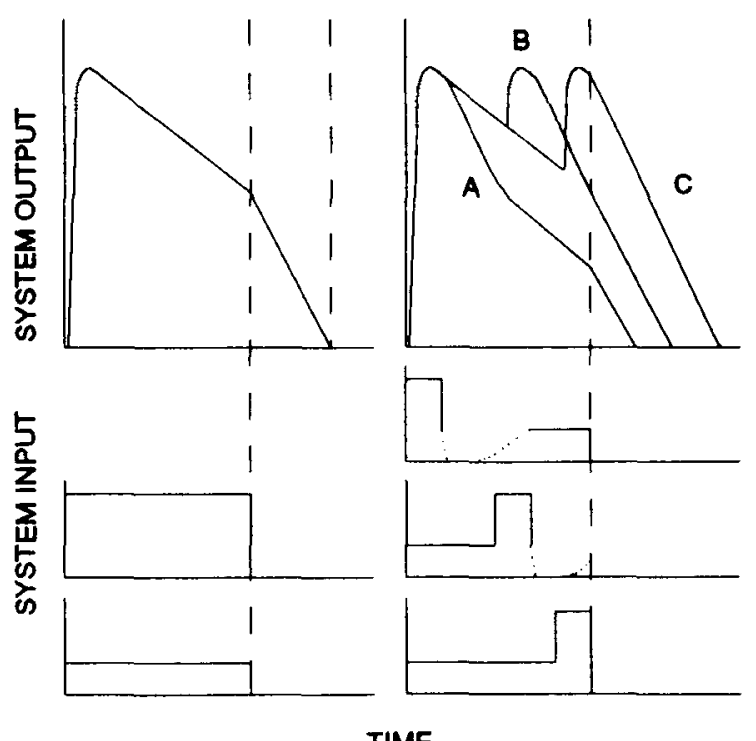

TIME

Figure 7. Schematic representation of input-output relationships for a hypothetical single-process feedhack-controlled mechanism of visible persistence. See text for explanation. 
present assumptions. This equation shows that, within the constraints of the model, persistence is an inverse linear function of stimulus duration. The model, then, is consistent with both the inverse duration effect and the high degree of linearity typically observed in empirical functions relating persistence to stimulus duration. In previous attempts to explain this pattern of results, it has been proposed that stimuli initiate activity of constant duration in the persistence mechanism, and consequently, a slope of approximately -1.0 has been predicted for the function relating persistence to stimulus duration. As mentioned above, Hogben and Di Lollo (1990) have consistently found this slope to be approximately -0.4 . The present model readily accommodates these findings in that the predicted slope of the function is not constrained to a specific theoretical value, but governed by the ratio of the rate of decrease in system output during stimulus presentation to the rate immediately following stimulus presentation-that is, by $m_{1} / m_{2}$.

The model also accounts for the principal finding from Experiment 3: that the persistence of stimuli that terminated with a 20-msec luminance increment was independent of the duration of the stimulus segment that preceded the increment. It need only be proposed that the onset of the luminance increment returned output to its maximum level, after which system output decreased as it would for a 20-msec stimulus. Had the level of the luminance increment been progressively reduced in Experiment 3, the model predicts that it would have eventually fallen below the level necessary for the luminance increment to restore output to its maximum level, and would thereafter have produced a graded decline in performance as the peak level to which output was actually raised converged on the level attained in the absence of a luminance increment. This is the result obtained by Di Lollo and Bourassa (1983, Experiment 4) in just such an experiment.

The one finding of the present study that cannot be accounted for without elaboration of this simple model is that the persistence of both the continuously incremented and the unincremented inducing stimuli examined in Experiment 1 was longer than that of the stimulus of equal duration that commenced with a $20-\mathrm{msec}$ luminance increment. Given that both the unincremented and incremented luminance levels have previously been inferred to hold the rate of decrease in system output to its minimum level, the presence of the brief luminance increment should have had no effect on the time response of the system. This apparent discrepancy may eventually be found to be predicted, given precise specification of the time response of the excitatory and inhibitory feedback components of the system response. However, we can offer a relatively simple explanation in terms of the model outlined: specifically, that the offset of the luminance increment was immediately followed by a transient reduction in system input to levels below those required to sustain the minimum rate of decrease in system output. Masking effects in which the presentation of a stimulus reduces the perceived luminance of stimuli at adjacent retinal locations are well documented (see, e.g., Alpern, 1953). A similar effect is suggested by the reduced visibility of a target when its retinal location is overlapped by a masking stimulus that precedes it by some brief interval (Kahneman, 1968).

The right panel of Figure 7 illustrates how transient masking of the stimulus segment that follows the luminance increment accounts for the reduction in persistence observed when the increment is placed at the onset of a long-lasting inducing stimulus. System input drops briefly below the level required to maintain minimum rates of decrease in system output, causing a brief drop in output to levels below those attained in the maximum system response, and leading, ultimately, to the earlier decrease of output to subthreshold levels. As the brief luminance increment is moved toward the end of the stimulus, the output level attained at the time corresponding to stimulus offset will increase and eventually equal that attained in the no-increment condition, thereby producing equal persistence. In Experiment 1, this occurred when the luminance increment followed stimulus onset by approximately $40 \mathrm{msec}$. When the luminance increment is moved much closer to the end of the stimulus, it will produce longer durations of persistence despite the decrement in output associated with its offset.

The proposition that the offset of the luminance increment is followed by a transient reduction in the level of system input is entirely ad hoc. However, it does lead immediately to at least one testable prediction. If the rate of decrease in system output is higher following a luminance increment than when luminance is constant, then Equation 1 implies that the gradient of the function relating the duration of persistence to stimulus duration must be steeper for stimuli that commence with a brief luminance increment than for simple rectangular pulses. As noted above, in the case of simple rectangular pulses, the gradient of the function relating ISI $_{50}$ to stimulus duration has been estimated as approximately -0.4 . By comparison, the stimuli that commenced with a 20 -msec luminance increment in Experiment 2 produced a corresponding gradient of approximately -0.75 for the 3 observers. This difference is in the expected direction and lends support to the proposition that the offset of the luminance increment was associated with a reduction in the level of system input.

With only slight elaboration, then, the model representing our first approximation to a single-process feedbackcontrolled mechanism accommodates all the relevant findings of the present study and is entirely consonant with findings in related studies.

When supplemented with a few relatively minor assumptions concerning the structure of the persistence mechanism, this model can also account for the spatial proximity effect detailed in the introduction. Preserving information concerning the spatial patterning of stimula- 
tion almost certainly involves spatial differentiation at the sensory input level. In other words, the persistence mechanism is likely to have a receptive field organization in which stimulation of any point on the retina produces activity in particular subunits of this mechanism: specifically, those whose receptive fields overlap at that point. The strength of persistence at a given retinal location (or probability that the summated activity of subunits representing that location will reach suprathreshold levels) will vary with the number of subunits that are responding to stimulation at that location. Our findings indicate that a luminance increment is able to commandeer subunits of the persistence mechanism that would otherwise continue to respond to preceding stimulus segments. It would follow that the persistence of a stimulus can be attenuated by a second stimulus that is presented anywhere within the receptive fields of subunits that are responding to that stimulus. As successively presented stimuli are moved closer together, the temporally trailing stimulus will impinge on a progressively larger number of the receptive fields that encompass the preceding stimulus, thereby producing progressively greater attenuation of the persistence of the preceding stimulus.

Even in the crude form outlined, a model of this kind has obvious heuristic value and, in our opinion, offers the most parsimonious account of the empirical behavior of the persistence mechanism that operates in the pattern integration task. We make no pretence that the model might offer an explanation of persistence in the sense of identifying specific responses of the visual system or particular physiological substrates with the principal functional properties of the mechanism proposed. However, it does provide a conceptual framework within which these properties can be explored in a manner that is largely unconstrained by speculation concerning the structural aspects of the mechanism.

Our representation of the mechanism underlying persistence has been quite deliberately framed in a manner that is amenable to mathematical modeling. This level of formalization forces us to address key issues in the interpretation of the data that have generally been overlookednot least among them the question of how the system responses to the two stimuli in a temporal integration task are combined to yield a graded level of performance. In particular, we might ask whether performance is proportional to the degree of temporal overlap between these responses or to some more complex parameter such as the ratio of the period of overlap (or conjunction) to the period of disjunction, as suggested by Groner, Bischof, and Di Lollo (1988).

The immediate challenge of this line of research is to produce a mathematical description of the simplest control system capable of behaving in the manner dictated by the data, not in the belief that the resulting model of the system will necessarily have a direct physiological counterpart, but simply in an effort to come to terms with the probable and necessary functional properties of this mechanism. The predictive capabilities of such a model may well prove worthwhile ends in themselves.

\section{REFERENCES}

ALPERN, M. (1953). Metacontrast. Joumal of the Optical Sociery of America, 43, 648-657.

ARDEN, G. B., WEALE, R. A. (1954). Variations of the latent period of vision. Proceedings of the Royal Society (London), Series B, 142, 258-266.

BAKER, H. D. (1963). Initial stages of dark and light adaptation. Journal of the Optical Society of America, 53, 98-103.

Bowling, A., Lovegrove, W. (1980). The effect of stimulus duration on the persistence of gratings. Perception \& Psychophysics, 27. 574-578.

BREITMEYER, B. (1984). Visual masking: An insegrative approach. Oxford, U.K.: Oxford University Press.

Clark, C. D., HogBen, J. H. (1990). Visible persistence and motion smear. Manuscript in preparation.

Coltheart, M. (1980). Iconic memory and visible persistence. Perception \& Psychophysics, 27, 183-228.

CrawFord, B. H. (1947). Visual adaptation in relation to brief conditioning stimuli. Proceedings of the Royal Sociery (London), Series B, 134, 283-302.

Di LoLLo, V. (1979). Luminous calibration of oscilloscopic displays. Behavior Research Methods \& Instrumentation, 11, 419-421.

Di LoLLo, V. (1980). Temporal integration in visual memory. Journal of Experimental Psychology: General, 109, 75-97.

Di Lollo, V., * Bourassa, C. M. (1983). Temporal integration following intensification of long-lasting visual displays. Vision Research, 23, 677-687.

Di lollo, V., Clark, C. D., Hogben, J. H. (1988). Separating visible persistence from retinal afterimages. Perception \& Psychophysics, 44, 363-368.

Di LolLo, V., FinleY, G. (1986). Equating the brightness of brief visual stimuli of unequal durations. Behavior Research Methods, Instruments, \& Computers, 18, 582-586.

Di Lollo, V., \& HocBen, J. H. (1985). Suppression of visible persistence. Joumal of Experimental Psychology: Human Perception \& Performance, 11, 304-316.

Di Lollo, V., \& Hogben, J. H. (1987). Suppression of visible persistence as a function of spatial separation between inducing stimuli. Perception \& Psychophysics, 41, 345-354.

Dixon, N. F., Hammond, J. (1972). The attenuation of visible persistence. British Journal of Psychology, 63, 243-254.

EFron, R. (1970a). The minimum duration of a perception. Neuropsychologia, 8, 57-63.

EFRON, R. (1970b). The relationship between the duration of a stimulus and the duration of a perception. Neuropsychologia, 8, 37-55.

EFroN, R., \&EE, D. N. (1971). The visual persistence of a moving stroboscopically illuminated object. American Joumal of Psychology, 84, 365-375.

FARRELL, J. M. (1984). Visible persistence of moving objects. Journal of Experimental Psychology: Human Perception \& Performance, 74, 476-484.

Groner, M. T., Bischof, W. T., \& Di Lollo, V. (1988). A model of visible persistence and temporal integration. Spatial Vision, 3 , 293-304.

Hogben, J. H., \& Di Lollo, V. (1974). Perceptual integration and perceptual segregation of brief visual stimuli. Vision Research, 14, 1195-1201.

Hogben, J. H., \& Di Lollo, V. (1985). Suppression of visible persistence in apparent motion. Perception \& Psychophysics, 38, 450-460.

HoGBeN, J. H., DI LoLLo, V. (1990). An examination of the inverse duration effect in temporal integration. Manuscript in preparation. 
KAHNEMAN, D. (1968). Method, findings and theory in studies of visual masking. Psychological Bulletin, 70, 404-425.

Kolers, P. A. (1972). Aspects of motion perception. Oxford, U.K.: Pergamon Press.

Lovegrove, W., Martin, F., \& Slaghuis, W. (1986). A theoretical and experimental case for a visual deficit in specific reading disability. Cognitive Neuropsychology, 3, 225-267.

NisLY, S. J., \& WASSERMAN, G. S. (1989). Intensity dependence of perceived duration: Data, theories, and neural integration. Psychological Bulletin, 106, 483-496.

Rours, J. A. J. (1963). Perception lag as a function of stimulus luminance. Vision Research, 3, 81-91.
Serviere, J., Micel, D., \& Glifret, Y. (1977a). Electrophysiological correlates of the visual perception of "instantaneous" and "durable." Vision Research, 17, 65-69.

Serviere, J., Miceli, D., \& Galifret, Y. (1977b). A psychophysical study of the visual perception of "instantaneous" and "durable." $V i$ sion Research, 17, 57-63.

TAylor, M. M., Creelman, C. D. (1967). PEST: Efficient estimates on probability functions. Journal of the Acoustical Society of America, 41, 782-787.

(Manuscript received September 8, 1989; revision accepted for publication September $5,1990$. ) 